\title{
EDITORIAL
}

\section{HACIA LA PREVENCIÓN Y EL CONTROL DEL TABAQUISMO EN ESPAÑA}

Ana Pastor Julián

Ministerio de Sanidad y Consumo.

Nos encontramos ante la oportunidad de establecer un marco común de actuación frente a uno de los principales problemas de Salud Pública, el tabaquismo, que representa la primera causa de enfermedad y muerte prevenible en nuestro país.

Contamos con un amplio consenso. En todo el territorio nacional se ha ido extendiendo progresivamente la necesidad de dar la respuesta adecuada a esta epidemia silenciosa de las sociedades desarrolladas, comenzando un movimiento de opinión, sensibilización y concienciación, liderado desde los distintos sectores técnicos, políticos, y cada vez más creciente, sociales.

Consecuentemente, se han ido desarrollando actividades encaminadas a la prevención y control del tabaquismo en toda nuestra geografía, desde Comunidades Autónomas, Ayuntamientos y Administración Central, asî como por Sociedades Científicas, Asociaciones Profesionales y Organizaciones Sociales.

El Plan Nacional de Prevención y Control del Tabaquismo que presentamos, trata de establecer el marco más adecuado para potenciar todas estas iniciativas que se vienen realizando, facilitar el desarrollo de otras nuevas, y difundir con amplitud y oportunidad, la información y los conocimientos, fortaleciendo así un cuerpo común que fomente una sociedad libre de tabaco y desarrolle las estrategias para alcanzar sus objetivos.
También tiene presente la complejidad y dificultades existentes en el control del tabaquismo, por lo que señala la necesidad de reunir todos los esfuerzos para conseguir un efecto sinérgico y multiplicador, compartiendo objetivos y un enfoque global del problema.

En esta misma línea, entendemos muy oportuno continuar participando en el horizonte marcado por las iniciativas internacionales. La Organización Mundial de la Salud contempla en la Estrategia Europea para el Control del Tabaquismo que los Estados miembros cuenten con planes propios, así como políticas globales, según se propone en el Convenio Marco para el Control del Tabaco. También desde la Unión Europea se establecen líneas de acción de interés y compromiso para España.

Así, desde la Presidencia del Consejo Interterritorial del Sistema Nacional de Salud, en su reunión de fecha 30 de junio de 2001, se presenta en líneas básicas, la propuesta de un Plan Nacional de Prevención y Control del Tabaquismo, para que sea elaborado conjuntamente por las Comunidades Autónomas y el Ministerio de Sanidad y Consumo.

Como acuerdo, se propone que dicho Plan Nacional sea elaborado por el Grupo de Trabajo de Tabaco, constituido en la Ponencia de Promoción de la Salud de la Comisión de 
Salud Pública, y formado por técnicos responsables en este tema de las Comunidades Autónomas y de la Subdirección General de Promoción de la Salud y Epidemiología del Departamento.

Con anterioridad, este Grupo de Trabajo de Tabaco cumplió su primer compromiso, elaborando el Informe sobre el Tabaco, donde se recogía la descripción de la situación actual de las políticas e intervenciones que las administraciones sanitarias del Estado español desarrollan ante el tabaquismo, y ofrecer unas Recomendaciones que pudieran orientar los programas de actuación para mejorar la situación.

El Informe sobre el Tabaco, presentado en la Comisión de Salud Pública en septiembre de 2000, recoge como Recomendación básica la elaboración de un Plan Nacional de carácter intersectorial, en la línea propuesta por la OMS.

El presente Plan Nacional de Prevención y Control del Tabaquismo, elaborado por el Grupo de Trabajo de Tabaco, pretende recoger aquellos aspectos fundamentales para el abordaje global del tabaquismo, desde una estructura lo menos compleja posible, con unos objetivos consensuados, alcanzables desde distintas estrategias. Tiene en cuenta las distintas situaciones y grados de desarrollo, planteando disminuir las desigualdades. Se dibuja como un plan dinámico, conociendo bien el punto de partida y sabiendo con claridad los objetivos que persigue, dispuesto a revisiones y nuevas propuestas.

Representa el marco nacional de actuación de una política de Gobierno, que desde el liderazgo sanitario, cuenta con otras Administraciones y Departamentos Ministeriales, con colectivos profesionales y con la participación social. Dispone una estructura que cuenta con amplia participación intersectorial, a la vez que un funcionamiento ágil y práctico.

Responde al compromiso del Ministerio de Sanidad y Consumo, disponiendo de recursos humanos y materiales propios para la gestión y desarrollo del Plan, y de las Comunidades y Ciudades Autónomas, que también dispondrán de los recursos específicos que estimen necesarios.

En cumplimiento del acuerdo del Consejo Interterritorial del Sistema Nacional de Salud, que dio su aprobación el 13 de enero de 2003, se presenta el Plan Nacional de Prevención y Control del Tabaquismo para ser desarrollado en el periodo 20032007. 